\title{
Genomic comparative analysis of the environmental Enterococcus mundtii against enterococcal representative species
}

\author{
Guillermo D Repizo ${ }^{1}$, Martín Espariz ${ }^{1}$, Víctor S Blancato ${ }^{1}$, Cristian A Suárez ${ }^{1}$, Luis Esteban² ${ }^{2}$ and Christian Magni ${ }^{*}$
}

\begin{abstract}
Background: Enterococcus mundtii is a yellow-pigmented microorganism rarely found in human infections. The draft genome sequence of E. mundtii was recently announced. Its genome encodes at least 2,589 genes and 57 RNAs, and 4 putative genomic islands have been detected. The objective of this study was to compare the genetic content of E. mundtii with respect to other enterococcal species and, more specifically, to identify genes coding for putative virulence traits present in enterococcal opportunistic pathogens.
\end{abstract}

Results: An in-depth mining of the annotated genome was performed in order to uncover the unique properties of this microorganism, which allowed us to detect a gene encoding the antimicrobial peptide mundticin among other relevant features. Moreover, in this study a comparative genomic analysis against commensal and pathogenic enterococcal species, for which genomic sequences have been released, was conducted for the first time. Furthermore, our study reveals significant similarities in gene content between this environmental isolate and the selected enterococci strains (sharing an "enterococcal gene core" of 805 CDS), which contributes to understand the persistence of this genus in different niches and also improves our knowledge about the genetics of this diverse group of microorganisms that includes environmental, commensal and opportunistic pathogens.

Conclusion: Although E. mundtii CRL1656 is phylogenetically closer to E. faecium, frequently responsible of nosocomial infections, this strain does not encode the most relevant relevant virulence factors found in the enterococcal clinical isolates and bioinformatic predictions indicate that it possesses the lowest number of putative pathogenic genes among the most representative enterococcal species. Accordingly, infection assays using the Galleria mellonella model confirmed its low virulence.

Keywords: Enterococcus, Enterococcus mundtii, Genomic comparative analysis

\section{Background}

The genus Enterococcus is a diverse group of low GC\% gram-positive bacteria that contains over 33 species. The genera include commensal species of the gastrointestinal tracts of humans and animals, and also environmental strains that can be isolated from soil, surface waters and plant material [1,2]. Enterococci are nutritionally fastidious microorganisms, which are associated with a large variety of human activities. In this sense, several strains

\footnotetext{
* Correspondence: magni@ibr-conicet.gov.ar

'Instituto de Biología Molecular y Celular de Rosario (IBR-CONICET) and Departamento de Microbiología, Facultad de Ciencias Bioquímicas y Farmacéuticas, Universidad Nacional de Rosario, Suipacha 531, Rosario S2002LRK, Argentina

Full list of author information is available at the end of the article
}

have technological relevance since they are present in dairy, meat and other fermented foods, and some of them show probiotic effects $[1,2]$. Nevertheless, they are not "generally recognized as safe" (GRAS) microorganisms for human consumption [U.S. Food Drug Administration (FDA) or European Food Safety Authority (EFSA)], even though they are phylogenetically related to the group of lactic acid bacteria (LAB). Particularly during the last decade, Enterococcus faecalis and Enterococcus faecium strains emerged as opportunistic human pathogens frequently associated with nosocomial infections with a high capacity to disseminate antibiotic resistance [3]. As a consequence, information on genetics and physiology of these species has increased dramatically in recent years; however, little data is available regarding other enterococci. 
Enterococcus mundtii was described as a non-motile, yellow-pigmented enterococcus typically isolated from plant material, soil, cow teats and milker's hands $[4,5]$ and infrequently associated to human infection [6]. As the rest of enterococci, they are facultative anaerobes and display a homolactic glucose metabolism. DNA GC content ranges from 38 to $39 \%$, as determined by the melting temperature method [4]. We have recently announced the draft genome sequence of E. mundtii strain CRL1656 [7]. This strain was isolated from stripping milk of an Argentinean cow and, given its bacteriocinogenic capacity, it has been proposed as a probiotic microorganism to prevent mastitis in these mammals [8].

During the last decade, the availability of bioinformatics approaches for comparison of multiple bacterial genomes allowed the analysis of a huge amount of sequence data. In this sense, the aim of the present study was to gain insight into the similarities between the genetic content of $E$. mundtii and other enterococcal species and, more specifically, to investigate genes coding for virulence factors shared with $E$. faecalis and $E$ faecium species, frequently behaving as opportunistic pathogens.

\section{Methods}

General inspection of the $E$. mundtii genome

Gene products putatively encoded by E. mundtii CRL1656 were identified using RAST [9] (GenBank accesion number: AFWZ00000000.1). In order to improve this sequence, a BLASTN approach (all versus all) was performed and those contigs shorter than 1,000 bp and with an homology higher than $99 \%$ with sequences already contained in a longer contig were deleted. In this manner, 87 contigs were removed from the database (approximately $200 \mathrm{~Kb}$ in total), resulting in a genome of $2.87 \mathrm{Mb}$ (GC content $38.4 \%)$. The remaining contigs were ordered and oriented with Advanced Pipmaker using E. faecalis V583 genome as a reference [10]. Finally, contigs were concatenated, using a Perl script designed ad hoc, by including the sequence NNNNNCACACACTTAATTA ATTAAGTGTGTGNNNNN, which harbors stop codons in all six reading frames [11].

After annotation of each of the draft genomic sequences in RAST (Table 1), as explained for E. mundtii, comparative genome analysis against other enterococcal species was performed. E. mundtii unique genes were determined by using RAST Compare Metabolic Reconstruction Tool [9].

\section{ANI versus shared genes with similar function plot}

ANI values were calculated based on pairwise alignment of genome stretches using the JSpecies software with BLAST algorithm [17]. Calculation of ANI values is implemented as described by Goris J, et al. [18]. Shared genes with similar function among each enterococcal species described in Table 1 and E. mundtii were assessed using the Compare Metabolic Reconstruction tool from RAST server. Genes which were associated within a RAST subsystem for both microorganisms were considered as shared between them [9].

\section{Study of genomic islands}

The analysis considered to detect putative Genome Islands (GEIs) was as follows. First, the regions detected by Alien-Hunter [19] were selected and secondly, positives results obtained either by Colombo/SIGI-HMM [20] or IslandPath-DIMOB [21] were taken as requirement for the next step. Regions identified using this approach were manually inspected using Artemis and DNAplotter [22] in order to find out further evidences related to GEIs, in general, and Pathogenic Island (PAIs), in particular [23]. A deviation in the $\mathrm{G}+\mathrm{C}$ content frequency (calculated by DNAPlotter) plus the presence of insertion sequences and tRNA flanking regions jointly with transposases coding genes, which are important for DNA incorporation processes, were accepted as further evidence of the presence of a GEI. Lastly, specific features of each gene located within the putative PAI were determined. Particularly, genes involved in virulence, antibiotic resistance, and pathogenic mechanisms were traced, using the BLASTP tool in the Virulence Factors Data Base server [24]. Others functions of the features found in these regions were extracted from RAST annotations.

\section{Phylogenetic tree construction}

This analysis involved sequences from nine enterococci species (Table 1) and the outgroup Lactococcus lactis SK11. Orthologous proteins were assigned using the OrthoMCL software [25]. In case that a particular enterococcal species contained more than one protein from the same group of orthologs, only the protein with the lower e-value was considered for the alignment. Concatenated orthologous protein sequences were aligned using ClustalX [26], and poorly aligned positions as well as excessively divergent regions were trimmed using GBlock 0.91b [27] resulting in a final alignment containing a total of 206,745 residues. Finally, the evolutionary history was inferred by using the Randomized Axelerated Maximum Likelihood algorithm (RAxML, [28]). DCMUT with empirical base frequencies and GAMMA distribution were used as substitution model. Reliability of the inferred tree was tested by bootstrapping with 1000 replicates.

\section{Analysis of regulators}

The presence of orthologs for putative regulator proteins of E. mundtii CRL1656 in E. faecalis V583 and E. faecium DO genomes were determined as described in Additional file 1: Figure S1. Briefly, all protein sequences 
Table 1 Enterococcal species analyzed in this study and relevant features of their genomic sequences

\begin{tabular}{|c|c|c|c|c|c|c|c|c|c|c|}
\hline Organism & BioProject & Status & $\begin{array}{l}\text { Size } \\
\text { (Mbp) }\end{array}$ & $\begin{array}{l}\mathrm{GC} \\
\%\end{array}$ & Genes & $\begin{array}{l}\text { Proteins } \\
\text { (PubMed) }\end{array}$ & $\begin{array}{l}\text { Proteins } \\
\text { (RAST) }\end{array}$ & $\begin{array}{l}\text { Putative pathogenicity } \\
\text { determinants }^{a}\end{array}$ & Source & References \\
\hline E. mundtii CRL1656 & PRJNA71221 & $\begin{array}{l}\text { Scaffolds or } \\
\text { contigs }\end{array}$ & 2.87 & 38.4 & 2,646 & 2,589 & 2,589 & 659 & Cow udder & [7] \\
\hline E. faecalis V583 & PRJNA57669, PRJNA70 & Complete & 3.36 & 37.4 & 3,412 & 3,264 & 3,363 & 1,006 & Clinical & [10] \\
\hline E. faecalis 62 & $\begin{array}{l}\text { PRJNA159663, } \\
\text { PRJNA61185 }\end{array}$ & Complete & 3.13 & 37.4 & 3,157 & 3,075 & 3,075 & 876 & Commensal & [12] \\
\hline E. faecium XT16 (DO) & $\begin{array}{l}\text { PRJNA55353, } \\
\text { PRJNA30627 }\end{array}$ & Complete & 3.05 & 37.9 & 3,209 & 3,114 & 2,779 & 728 & Clinical & [13] \\
\hline E. faecium Com15 & $\begin{array}{l}\text { PRJNA55725, } \\
\text { PRJNA32967 }\end{array}$ & $\begin{array}{l}\text { Scaffolds or } \\
\text { contigs }\end{array}$ & 2.77 & 38.2 & 2,783 & 2,724 & 2,773 & 755 & Commensal & [14] \\
\hline E. italicus DSM 15952 & $\begin{array}{l}\text { PRJNA61487, } \\
\text { PRJNA53039 }\end{array}$ & $\begin{array}{l}\text { Scaffolds or } \\
\text { contigs }\end{array}$ & 2.31 & 39.2 & 2,455 & 2,405 & 2,275 & 700 & Cheese & [15] \\
\hline $\begin{array}{l}\text { E. casseliflavus ATCC } \\
12755\end{array}$ & $\begin{array}{l}\text { PRJNA63559, } \\
\text { PRJNA53041 }\end{array}$ & $\begin{array}{l}\text { Scaffolds or } \\
\text { contigs }\end{array}$ & 3.55 & 42.4 & 3,606 & 3,548 & 3,415 & 942 & $\begin{array}{c}\text { Oral } \\
\text { commensal }\end{array}$ & [14] \\
\hline E. gallinarum EG2 & $\begin{array}{l}\text { PRJNA55685, } \\
\text { PRJNA32927 }\end{array}$ & $\begin{array}{l}\text { Scaffolds or } \\
\text { contigs }\end{array}$ & 3.13 & 40.6 & 3,079 & 3,041 & 3,072 & 862 & Clinical & [16] \\
\hline $\begin{array}{l}\text { E. saccharolyticus } \\
\text { ATCC } 43076\end{array}$ & $\begin{array}{l}\text { PRJNA206365, } \\
\text { PRJNA191890 }\end{array}$ & $\begin{array}{l}\text { Scaffolds or } \\
\text { contigs }\end{array}$ & 2.60 & 36.9 & 2,625 & 2,582 & 2,594 & 780 & $\begin{array}{l}\text { Straw } \\
\text { bedding }\end{array}$ & [15] \\
\hline
\end{tabular}


assigned as regulator proteins by RAST were used as query in a BLASTP search over E. faecalis V583 and E. faecium DO genome sequences. Cut-Off and Cut-In values were established as described in Additional file 1: Figure S1C. Then, E. faecalis V583 and E. faecium DO proteins sharing an identity percentage with $E$. mundtii CRL1656 proteins higher than the Cut-In value were considered as present in the E. mundtii genome while those with lower identity percentages were considered absent. Proteins with shared identity between Cut-In and Cut-Off values were further individually analyzed according to their gene context (Additional file 1: Figure S1D). For those E. mundtii CRL1656 proteins showing no homologs in E. faecium or E. faecalis a BLASTP was performed against a non-redundant database (RefSeq and Genbank) with a bit-score cut-off of 60 .

Semiqualitative comparison of potential virulence factors For the enterococcal species described in Table 1, BLASTP analysis of their respective proteomes was made against the Virulence Factors Data Base [24]. The expectation value used as cut-off was $10^{-10}$. Only the first hit was recovered for each protein query.

\section{Galleria mellonella killing assay}

Infection of Galleria mellonella larvae with E. faecalis was accomplished as previously described by Lebreton et al. [29]. Briefly, using a syringe pump (microliter \#750, Hamilton), larvae (about $0.2 \mathrm{~g}$ and $3 \mathrm{~cm}$ in length) were infected subcutaneously with washed E. faecalis JH2-2 or E. mundtii CRL 1656 cells from an exponential culture in LBG administered in $5 \mu \mathrm{l}$ of sterile saline buffer. Control groups of larvae received $5 \mu$ l of a saline solution only. In each test, 15 larvae were infected and the experiments were repeated at least three times. Larval killing was monitored up to 89 hours post-infection. Survival curves were constructed by the Kaplan-Meier method and compared by Log-rank analysis ( $\mathrm{R}$ statistical software). $\mathrm{P}$ values of $<0.05$ were considered statistically significant [29].

\section{Results}

\section{General features of the $E$. mundtii genome and phylogenetic analysis}

The genome sequence of E. mundtii strain CRL1656 was automatically annotated by using the RAST server [9]. A total of 2,589 coding sequences (CDS) and 57 structural RNAs (52 tRNAs) were predicted by this method. Putative biological roles have been assigned for 1,724 (67\%) of the ORFs, whereas the remaining 865 (33\%) encode hypothetical proteins for which no probable function could be predicted. The general features of the genome are summarized in Figure 1 and Table 1.
E. mundtii CRL1656 phylogeny was analyzed with a tree generated from the concatenated sequences of 805 core proteins from nine enterococci and the outgroup L. lactis SK11 (Figure 2A). In this analysis we included representative enterococcal species of diverse origins, whose genomic sequences have been released (Table 1). We observed that E. mundtii is more related to $E$. faecium, which is frequently associated with nosocomial infections.

To further analyze the relationships among these enterococcal species, we constructed a plot of the shared genes with similar function between $E$. mundtii and the species under comparison versus average nucleotide identity (ANI) values (Figure $2 \mathrm{~B}$ ). ANI values are frequently used to verify prokaryotic species definitions $[18,30]$. As expected, these values were lower than the 94\% accepted as a threshold for species designation [18,30]. E. faecium strains showed the highest ANI values (around 76\%), with a content of shared genes with similar function between 44 and $46 \%$. With respect to other enterococcal species, ANI varies within a narrow range (approximately 71\%) and E. mundtii shared more genes with similar function with strains of non-clinical origin as E. casseliflavus ATCC12755 and E. saccharolyticus ATCC 43076 than the rest of the analyzed strains.

We were also interested in performing a bioinformatic quantification of genes horizontally transferred to the $E$. mundtii genome. According to the Alien-Hunter software, $39 \%$ of the genome was acquired by horizontal transfer. A list of genes coding for integrases, transposases and phage-related proteins found by the RAST annotation is given in Additional file 2: Table S1. In this regard, four gene clusters that fit the criteria as GEIs (refer to Methods for details) were detected and are pointed out in Figure 1. GEI I contains a putative cell wall surface anchor protein, that could be involved in adhesion and invasion mechanisms. Interestingly, a gene for the bacteriocin mundticin was detected in the GEI III cluster, which is genetically linked to its immunity protein and transporter.

Regarding genetically distinctive features of $E$. mundtii, a comparative analysis performed between strain CRL1656 and the other enterococcal species included in this study (Table 1) revealed a set of 22 unique proteins with putative functions for this microorganism (Table 2). Among this group of proteins, besides the mundticin previously mentioned, two proteins that could be responsible for capsule biosynthesis (see Virulence factors and antibiotic resistance section) and a toxin-antitoxin MazE-MazF system were detected.

\section{Identification of a gene encoding a putative bacteriocin} A cluster of three genes involved in bacteriocin synthesis and transport, which is unique to this microorganism, 


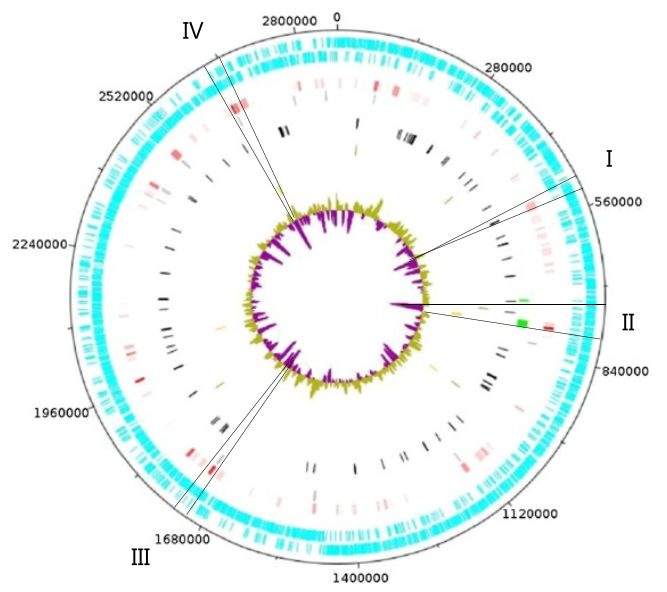

Genes with putative function located

in each of the predicted Genomic Islands (GEI)

GEI I:

Cell wall surface protein $2 \mathrm{C}$

GEI II:

LSU ribosomal protein L35p

LSU ribosomal protein L20p

Retron-type RNA-directed DNA polymerase

Phage integrase

Resolvase/integrase

Major Facilitator Superfamily

GEI III:

Resolvase like protein

Recombinase sin

Piscicolin 126 immun

Piscicolin 126 inmunity protein

Xacteriocin mundticin

Xanthine/uracil/thiamine/ascorbate permease

Cation-transporting ATPase

GEI IV:

SSU ribosomal $\mathrm{S} 9 \mathrm{p}$

DNA-cytosine methyltransferase

DNA-cytosine methyltransferase

Figure 1 Circular representation of the E. mundtii genome. Starting from the outer circle: Rings 1 and 2 (ligth-blue) indicate positions of protein-coding genes on positive and negative strands, respectively. Ring 3 (red) shows presumably foreign DNA as predicted by Alien Hunter. Ring 4 (grey) shows alien genes predicted by Columbo Sigi. Ring 5 (green) corresponds to IslandPath-DIMOB predictions. Ring 6 (black) shows positions of tRNA genes. Ring 7 (blue), putative tranposases. Ring 8 (ochre), integrases. Ring 9 represents the $\mathrm{G}+\mathrm{C}$ percentage, colored yellow for regions above median GC score (38\%) and violet for regions less than or equal to the median. Circular sectors I-IV highlight the position of putative genomic islands. A list of genes with putative functions in each GEl is presented.

was found. These genes were designated $\operatorname{mun} A, \operatorname{mun} B$, and munC. The structural gene $m u n A$ encodes a 58 -aminoacid mundticin precursor. The mature peptide is a class IIa mundticin with an estimated molecular weight of 4,289 Da and $\mathrm{pI}$ of 9.45. The BLASTP protein database homology search on the 43-amino acid deduced mature peptide, mundticin 1656, revealed that its sequence is identical to that of the plasmid encoded-mundticin KS from $E$. mundtii NFRI7393 [31] and a 95\% identical to that of E. mundtii ATO6 [32]. The pre-peptide contains a leader peptide of 15 amino acids with a consensus GGXaa processing site. The mature peptide contains the YGNGV motif at positions $3-7$, characteristic of class IIa bacteriocins. Moreover, it contains the two cysteine residues (C-9 and C-14) forming the disulfide bridge, which are well conserved in all class IIa bacteriocins [33].

The munB gene encodes a 674-amino acid polypeptide, which exhibits $98 \%$ of similarity with the 674amino acid ABC transporter MunB found in E. mundtii NFRI7393, involved in the maturation and excretion of
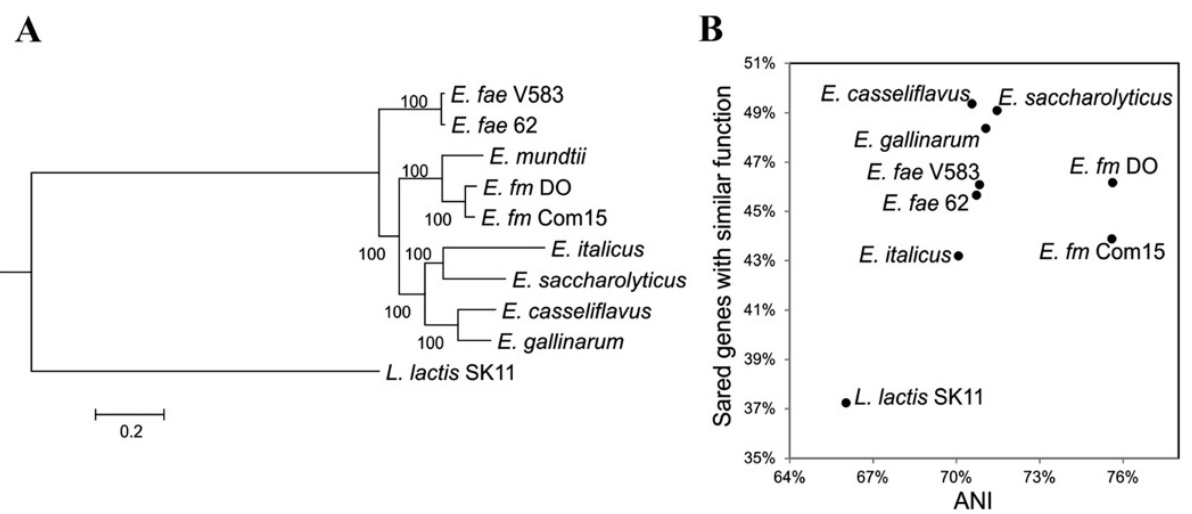

Figure 2 Phylogenetic analysis of E. mundtii CRL1656. (A) Core gene tree. ClustalX aligned sequences of core concatenated proteins were used for the phylogeny reconstruction analysis of enterococci species using the Randomized Axelerated Maximum Likelihood (RAxML) algorithm based on DCMUT with empirical base frequencies and GAMMA distribution model. The reliability of the inferred tree was tested by bootstrapping with 1000 replicates. The tree with the highest log likelihood (-2038246) is shown. Lactococcus lactis SK11 was included as outgroup species. (B) ANI plot. Shared genes with similar function and ANI values between E. mundtii and the bacterial genomes of the indicated strains are plot. E. mundtii CRL1656 (E. mundtii), L. lactis subsp. cremoris SK11 (L. lactis), E. italicus DSM15952 (E. italicus), E. casseliflavus ATCC12755 (E. casseliflavus), E. faecalis 62 (E. fae 62), E. faecalis V583 (E. fae V583), E. saccharolyticus ATCC 43076 (E. saccharolyticus), E. gallinarum EG2 (E. gallinarum), E. faecium Com15 (E. fm Com15), and E. faecium DO (E. fm DO). The data set supporting the results of this article is available in the TreeBASE repository, [http://purl.org/phylo/treebase/phylows/study/TB2:S15854]. 
Table 2 Unique genes among enterococcal species found in the $E$. mundtii genome

\begin{tabular}{|c|c|c|c|c|c|}
\hline Feature ID & Start & Stop & Length (AA) & Putative function & GEI \\
\hline fig|6666666.25220.peg.49 & 39017 & 39349 & 111 & Putative EsaC protein analog (Listeria type 3) & \\
\hline fig|6666666.25220.peg.389 & 768326 & 768559 & 78 & Programmed cell death antitoxin MazE & \\
\hline fig|6666666.25220.peg.401 & 780662 & 782068 & 469 & Retron-type RNA-directed DNA polymerase (EC 2.7.7.49) & \\
\hline fig|6666666.25220.peg.507 & 875260 & 875538 & 93 & Zinc finger domain-containing protein & \\
\hline fig|6666666.25220.peg.523 & 889680 & 889808 & 43 & Probable GTPase related to EngC & \\
\hline fig|6666666.25220.peg.751 & 1130121 & 1131056 & 312 & Mtx2 & \\
\hline fig|6666666.25220.peg.781 & 1159802 & 1160566 & 255 & Hnh endonuclease & \\
\hline fig|6666666.25220.peg.928 & 1322901 & 1323290 & 130 & Holin & \\
\hline fig|6666666.25220.peg.1128 & 1488458 & 1487922 & 179 & Rhs-family protein & \\
\hline fig|6666666.25220.peg.1403 & 1721023 & 1720727 & 99 & putative piscicolin 126 immunity protein & III \\
\hline fig|6666666.25220.peg.1405 & 1723390 & 1723214 & 59 & Bacteriocin mundticin & III \\
\hline fig|6666666.25220.peg.1456 & 1777938 & 1778441 & 168 & Phosphoribosylanthranilate isomerase like (EC 5.3.1.24) & \\
\hline fig|6666666.25220.peg.1618 & 1965831 & 1965595 & 79 & COG0477: Permeases of the major facilitator superfamily & \\
\hline fig|6666666.25220.peg.1862 & 2217937 & 2219130 & 398 & ATPase & \\
\hline fig|6666666.25220.peg.1907 & 243734 & 245122 & 463 & endo-beta-galactosidase, GlcNAc-alpha-1,4-Gal-releasing & \\
\hline fig|6666666.25220.peg.1961 & 2301444 & 2302463 & 340 & PE-PGRS family protein & \\
\hline fig|6666666.25220.peg.2071 & 2406250 & 2405168 & 361 & capsular polysaccharide biosynthesis protein $\mathrm{Cps} 4 \mathrm{G}$ & \\
\hline fig|6666666.25220.peg.2073 & 2408118 & 2407363 & 252 & putative capsule biosynthesis protein & \\
\hline fig|6666666.25220.peg.2156 & 2483290 & 2482904 & 129 & Putative EsaC protein analog (Listeria type 3) & \\
\hline fig|6666666.25220.peg.2203 & 2515077 & 2514832 & 82 & SMT0609 replicon stabilization protein (antitoxin to SMT0608) & \\
\hline fig|6666666.25220.peg.2352 & 2693714 & 2693941 & 76 & COG0477: Permeases of the major facilitator superfamily & \\
\hline fig|6666666.25220.peg.2403 & 2758856 & 2757387 & 490 & Internalin-like/N-acetylmuramoyl-L-alanine amidase & \\
\hline
\end{tabular}

enterocin KS [31]. The munC gene encodes a mundticin immunity protein of 98 amino acids, as evidenced by the close similarity to the immunity protein of mundticin KS [31].

\section{Stress response systems}

One of the most relevant characteristics of the Enterococcus genus is its capacity to resist and grow at low $\mathrm{pH}$. Thus, we analyzed the presence of genes involved in the acidic stress response in the $E$. mundtii genome. We identified eight genes encoding the different subunits of the $\mathrm{F}_{0} \mathrm{~F}_{1}$-ATPase, which is also present in the enterococcal core genome previously described. This complex is involved in $\mathrm{pH}$ homeostasis through the generation of proton motive force by ATP consumption [34]. The organization of these genes was the same as that for other enterococcal $\mathrm{F}_{0} \mathrm{~F}_{1}$-ATPases (atpBEFHAGDC), where atpBEF encode the $\mathrm{A}, \mathrm{C}$ and $\mathrm{B}$ subunits of the membrane-bound $\mathrm{F}_{0}$ domain, whereas atpHAGDC genes encode the $\delta, \alpha, \gamma, \beta$ y $\varepsilon$ subunits of the cytoplasmic $\mathrm{F}_{1}$ domain, respectively [34]. In addition, the genome of E. mundtii possesses an operon constituted by nine genes (F, I, K, E, C, G, A, B and D) homologous to the $n t p$ genes of $E$. hirae [35] encoding a $\mathrm{Na}^{+}$-pumping $\mathrm{V}_{1} \mathrm{~V}_{0}$-ATPase. The $\mathrm{V}_{1}$ domain is a peripheral complex responsible for ATP hydrolysis, and is constituted by A, $B, C, D, E, F$ and $G$ subunits. The $V_{0}$ domain is an integral complex responsible for $\mathrm{Na}^{+}$translocation across the membrane.

Furthermore, three gene clusters also indirectly involved in resistance to low $\mathrm{pH}$ were identified. One of them corresponds to the cit genes, homologous to those recently characterized in E faecalis, which are responsible for the degradation of citrate and are organized in two divergent putative operons [36-39]. The first transcriptional unit contains genes encoding a GntR-family transcriptional regulator and a citrate permease belonging to the $\mathrm{Me}^{2+}$-CitMHS family (citO and citH genes, respectively). In the second operon, genes responsible for the codification of citrate lyase subunits (citDEF), citrate lyase accessory genes ( $\operatorname{cit} C$ and $\operatorname{cit} X)$ and a membrane oxaloacetate decarboxylase (oadAHBD) were found. This genetic organization resembles that found in E. faecium, which differs from the E. faecalis cit locus in the position of another citrate lyase-accesory gene, citG, and also in the absence of the soluble oxaloacetate decarboxylase gene, citM. The second group of genes involves the mleS (malolactic enzyme), mleT (malate transporter) and mleR (transcriptional activator from LysR family) genes, which are required for the malolactic fermentation and show 
homology to those found in E. faecium [40]. Finally, genes associated to the arginine deiminase pathway (ADI), which generates ATP and contributes to low-pH resistance, were identified. These genes are present in the core genome of selected enterococci and code for the three main enzymes of the system, arginine deiminase, ornithine transcarbamylase and carbamate kinase. Additionally, we identified a homologue to $\operatorname{arcR}$ divergently oriented, which encodes an activator of the ADI system (Crp-Fnr family of regulators). Besides, a homologue of the arginine-ornithine transporter $(\operatorname{arcD})$ of Lactococcus garvieae ATCC 49156 was identified in another genomic region of $E$. mundtii.

Other related proteins required for optimal stress resistance were identified in the genome of $E$ muntdii: RecA, a mediator of homologous recombination and regulator of the SOS response, chaperonins GroEL and GroES, HtrA, a protein involved in proteolysis of abnormal proteins synthesized under stressful conditions, the enzymes involved in the synthesis of D-alanyl-lipoteichoic acid (dlt operon, see Table 3), and the diacylglycerol kinase DagK, involved in acid resistance [34].

\section{Analysis of regulators}

Since adaptation to different niches requires a finetuning of gene expression, we searched for regulators responsible for sensing the bacterial environmental milieu and physiological state. By knowing the set of regulators shared by related bacteria and detecting the presence of those that are unique, it is possible to infer the common and specific characteristics of niches and lifestyle among them. In the attempt to study the complete set of regulators encoded by the E. mundtii genome, a database consisting of 122 genes was constructed (see Methods section) and a comparative analysis against E. faecalis V583 and E. faecium DO was performed.

\section{Core regulators}

$58(48 \%)$ of the analyzed genes were found in the three genomes (Additional file 1: Figure S1). Although 24 of these genes are not assigned to any RAST subsystems, the rest of them were implied in diverse functions including sugar uptake and utilization (mannose, lactose, galactose, D-tagatose and galactitol), anabolic pathways

Table 3 Putative virulence factors present in $E$. mundtii

\begin{tabular}{|c|c|c|c|c|}
\hline Virulence factor & Genes & Homologous locus & Role in pathogenesis & Reference \\
\hline \multicolumn{5}{|l|}{ Adhesin } \\
\hline & $\mathrm{scm}$ & EfaeDRAFT_0418 & Adherence & [41] \\
\hline \multicolumn{5}{|l|}{ Pili } \\
\hline & $e b p A B C$ & EF1091-EF1093 & Biofilm formation & {$[42]$} \\
\hline & $e b p R$ & EF1090 & ebp locus regulation & [43] \\
\hline & strc & EF0194 & Pilus Sortase C & [42] \\
\hline & strA & EF3056 & Pilus Sortase A & [44] \\
\hline & $r n j B$ & EF1185 & ebp locus regulation & {$[45]$} \\
\hline \multicolumn{5}{|l|}{ Capsule } \\
\hline & eps locus & EFSG_00424, $-25,-26,-31,-33,-35$ to -41 & Resistance to phagocytosis & [16] \\
\hline \multicolumn{5}{|l|}{ Cell wall } \\
\hline & gall & EF1746 & Resistance to multiple types of stress & [46] \\
\hline & $\operatorname{bgs} A B$ & EF2891- EF2890 & Adherence and biofilm formation & [47] \\
\hline & $d l t$ locus & EF2749-EF2746 & Biofilm formation & {$[46]$} \\
\hline & bopD & EF0954 (MalR) & Biofilm formation & [48] \\
\hline & epa locus & EFWG_01395-EFWG_01370 & Biofilm and resistance to PMNs & [49] \\
\hline & $\operatorname{sag} A$ & EfaeDRAFT_1606 & Adherence & {$[50]$} \\
\hline \multicolumn{5}{|l|}{ Others } \\
\hline & efafm & EfaeDRAFT_2037 & Adherence & {$[43]$} \\
\hline & $m s r A$ & EF1681 & Oxidative stress & [51] \\
\hline & $m s r B$ & EF3164 & Oxidative stress & {$[51]$} \\
\hline & $\mathrm{g} / \mathrm{s} 33$ & EfaeDRAFT_2384 & Stress response protein & {$[52]$} \\
\hline & $\mathrm{g} / \mathrm{s} 20$ & EfaeDRAFT_2389 & Stress response protein & [52] \\
\hline & sigV1 and sigV2 & EF3180 & Mouse bacteremia & [53] \\
\hline & eep & EF2380 & Biofilm formation & [54] \\
\hline
\end{tabular}


(purine, pyrimidine, deoxyribonucleotides, glutamine, glutamate, aspartate and asparagine, fatty acids and pyridoxin), amine compounds degradation (arginine, ornithine, polyamine, chitin and $\mathrm{N}$-acetylglucosamine), ATP-dependent proteolysis, phosphate uptake, competence, heme and hemin uptake and utilization systems, metal homeostasis/resistance (copper, cobalt, zinc and cadmium) and oxidative stress (Additional file 3: Table S2, Core regulators).

\section{Non-core regulators}

37 genes (30\%) of the analyzed regulators were found in E. mundtii and E. faecium (20\%; 25 genes) or E. faecalis (10\%; 12 genes) but not in the three genomes at the same time (Additional file 1: Figure S1). Although higher cut-in and cut-off values were used for ortholog assignment to E. faecium with respect to E. faecalis (refer to Methods section), higher numbers of orthologs were found in the former, which correlates with the closer phylogenetic relationship between the species. While 25 out of the 37 genes are not assigned to any RAST subsystems, the remaining 12 were assigned to diverse functions including sugar uptake and utilization (fructooligosaccharides, raffinose, lactose and galactose), heme and hemin uptake and utilization systems, degradation of amine compounds (arginine and ornithine), organic acid metabolism (pyruvate and sialic acid) and oxidative stress (Additional file 3: Table S2. Non-core regulators).

\section{E. mundtii species-specific regulators}

27 gene orthologs (22\%) could not be found in E. faecium or E. faecalis, and $12(10 \%)$ of these genes have orthologs in other LAB. The remaining genes only have orthologs in phylogenetically more distant bacteria (Bacillus, Clostridium, Listeria). Although none of these genes belong to a RAST subsystem, a preliminary sinteny analysis assigned them to functions related to carbon compound utilization (maltose, xylose, cellobiose and formaldehyde), anabolic pathways (sulfur containing compounds, coenzyme A, lysine), cell surface functions, $\mathrm{ABC}$-type transporters and other roles (hydrolase, DNA methylation, ribosomal related) (Additional file 3: Table S2).

Among all the transcriptional regulators found in the E. mundtii genome, there is a subset corresponding to response regulators (RR) putatively forming part of twocomponent signal transduction systems (TCS). The importance of TCS for the ability of E. faecalis to respond to environmental stimuli has been previously stressed by Hancock and Perego [55]. The E. mundtii genome contains putative genes coding for 17 TCS and one orphan response regulator (Additional file 4: Table S3). We found E. faecalis homologues for 14 of the E. mundtii TCS and also for the orphan RR. As a general rule for most bacterial sensor kinases, all of the HK in E. mundtii were predicted to be membrane localized (data not shown). Among the remaining HK, one (IIb) showed homology to proteins present in environmental enterococci, and HKs IIIa-i and j presented homology to sensors encoded by E. faecium. This analysis suggests that the set of TCS present in E. mundtii is highly similar to that encoded by other enterococcal species.

\section{Identification of genes involved in pigmentation}

E. mundtti CRL1656 shows a characteristic yellowish pigmentation on agar plates, originated by the production of carotenoids as reported for other Enterococcus species [56]. Genes responsible for carotenoid production have been previously described in Staphylococcus aureus [57] and Lactobacillus plantarum [58]. It has been shown that $\operatorname{crt} M$ and $\operatorname{crtN}$ constitute a bicistronic operon encoding the enzymes responsible for the production of the yellow pigment, presumably the C30 carotenoid 4,4'-diaponeurosporene. In $S$. aureus, diaponeurosporene is further converted to staphyloxanthin, the orange carotenoid present in most staphylococci strains. For this, S. aureus harbors up to three extra enzymes coded by genes $\operatorname{crtO}$, crtP and crtQ, which are located in the same operon as $\operatorname{crtM}$ and $\operatorname{crtN}(\operatorname{crtOPQMN)}$ [57]. The E. mundtii genome encodes a protein with 68 and $77 \%$ similarity to $\mathrm{CrtN}$ from $S$. aureus and L. plantarum, respectively. Regarding CrtM, the homology is reversed, with the homologue from L. plantarum sharing a similarity higher than the one corresponding to S. aureus (64 versus 53\%, respectively). Remarkably, this analysis also detected the presence of putative homologues for S. aureus $\mathrm{CrtO}$ (60\%), CrtP (73\%) and CrtQ (52\%). However, genes coding for these proteins are located in a different cluster with respect to $\operatorname{crt} N$ and $\operatorname{crtM}$, and also show a different organization (crtPQO) to that found in $S$. aureus [57]. Furthermore, a comparative analysis with other enterococcal species indicated that $\operatorname{crt} N$ and $\operatorname{crt} M$ homologues were present in E. gallinarum, E. saccharolyticus and E. casseliflavius genomes. Noteworthy, putative proteins involved in staphyloxanthin biosynthesis were also detected in E. casseliflavius.

\section{Virulence factors and antibiotic resistance}

The potential virulence genes encoded by the genome of E. mundtii are of particular interest. An initial comparative analysis between different enterococcal species using the Virulence Factors Data Base [24] revealed that E. mundtii has a reduced number of putative virulence determinants (Table 1). We searched for virulence genes previously analyzed by G. mellonella or mice infection models $[3,46,59]$ to validate our results (Table 3). Among collagen adhesins, only the E. faecium widely spread $\mathrm{scm}$ gene [41] was detected in the E. mundtii genome, which lacks the most extensively studied ace and $\mathrm{acm}$ genes. We also found the $e b p$ pili coding cluster, in conjunction with its cognate transcriptional regulator EbpR [43] 
along with sortase proteins $[42,44]$, which are ubiquitous in E. faecalis and E. faecium. Furthermore, we identified the $r_{n j} B$ gene, which encodes a putative RNase J2 that activates the transcriptional expression of the ebpABC operon [45]. Noteworthy, expression of the $e b p$ locus is negatively regulated by the Fsr system [60], which is also present in E. mundtii (Additional file 4: Table S3). None of the other pili-encoding loci previously reported for E. faecalis (bee plasmidic genes, [61]) or E. faecium [41] were detected. A cluster of genes that might be responsible for the synthesis of capsular polysaccharides (eps locus, Additional file 5: Figure S2) was identified. It codes for proteins with homology to that reported in E. faecium [16]. In E. mundtii, we found three of the four phosphoregulatory system proteins conserved in all species of E. faecium (the LytR-Csp-Psr family protein is absent). Furthermore, two glycosyl transferases and the conserved dehydrogenase and flippase were detected as well as several conserved PTS-related proteins linked to the eps cluster in E. faecium 1,141,733 (Additional file 6: Table S4).

Moreover, several virulence genes that are related to cell wall synthesis in E. faecalis were found (Table 3). Remarkably, genes responsible for the synthesis of Epa (enterococcal polysaccharide antigen, suggested to be associated to the cell wall) were detected (Additional file 5: Figure S2). The core genes showed a similar genetic organization to that observed in E. faecium [16]. In $E$. mundtii the variable accessory region encode predicted glycosyltransferases and other proteins with potential roles in WTA production (encoded by tag genes), and the genetic organization resembles that of $E$. faecium Com15, a microorganism of human intestinal origin (Additional file 6: Table S4). Since major growth defects have been detected for mutants in different genes of this locus, Rigottier et al. [46] have proposed that these genes are important for the fitness of the bacterium rather than bona fide virulence factors.

Other virulence genes detected correspond to $\mathrm{msrA}$ and $m s r B$, encoding methionine sulfoxide reductases important for the oxidative stress response, macrophage survival, and persistent infection [51]; and $g l s$ genes encoding general stress proteins (Gls33 and Gls20), important for adaptation to the intestinal environment and in mouse peritonitis models [52]. Moreover, this analysis detected the presence of a gene with significant homology to bopD, coding for the transcriptional regulator of maltose metabolism, which has been implicated in biofilm formation and bacteremia in mice [48].

Regarding resistance to antibiotics, the search for vancomycin resistance genes (vanA, vanB, vanC, vanD and $v a n E)$, tetracycline (tet $M$, tet $L$, tetS, tet $O$, tet $K$, and tet $W$ ) gentamicin (aac (6')-aph (2')), chloramphenicol (cat), lincosamide $(\ln u A)$, erythromycin $(\operatorname{erm} T$ and
ermB), methicillin (mecA) and penicillin (blaZ) did not identify genes with significant homology (Additional file 7: Table S5). Furthermore, mutations conferring resistance to ampicillin or ciprofloxacin within the genes coding for gyrA or $p b p 5$, respectively, were neither identified.

\section{E. mundtii effect on G. mellonella survival}

In order to study E. mundtii virulence, CRL1656 strain was used to infect the insect host model Galleria mellonella $[29,46]$. G. mellonella, is a reliable model host to study the pathogenesis of numerous human pathogens [62]. The capacity of a pathogen to kill G. mellonella has a correlation with virulence in mammalian models [62]. In fact, the innate immune systems of Galleria larvae and mammals share a high degree of homology [63].

In our analysis, the $E$. faecalis $\mathrm{JH} 2-2$ strain was used as control [64]. This strain was initially isolated from a nosocomial infection, and constitutes a genetic model extensively used in our laboratory $[36,38,40,65]$ and by others $[66,67]$. The semiquantitative analysis performed (as described in the methods section) on the predicted proteins of the JH2-2 genome revealed 874 hits for putative virulence factors). As shown in Figure 3, G. mellonella survival was significantly LogRank test, $\mathrm{P}<0.05$ greater after infection with $E$. mundtii than with $E$. faecalis JH2-2 strain, at two different enterococcal concentrations. In the control groups infected with sterile saline buffered solution no larvae died in any of the replicates (data not shown). As consequence, these results confirm that E. mundtii is not as effective as E. faecalis in colonizing and killing G. mellonella larvae.

\section{Conclusion}

In this report, a study of the genomic data of E. mundtii CRL1656 is presented for the first time. Additionally, a comparative analysis including the full genomic sequence of representative enterococcal species of diverse origins was conducted (Table 1). E. mundtii CRL1656 contains 805 CDS in common with other species of Enterococcus and only a low number of unique CDS, among which we identified a cluster encoding a bacteriocin and other related proteins. A phylogenetic tree for concatenated sequences of enterococcal core proteins was constructed (Figure 2A), indicating that E. mundtii is closer to E. faecium. This is in accordance with the sodA gene sequence phylogenetic comparison reported by Poyart et al. [68], which showed that these two enterococcal species cluster together. Moreover, as shown in Figure 2B, the highest ANI percentage was found between these microorganisms.

Bacterial genome plasticity is influenced by the presence of GEIs, which may include genes encoding for any number of functions, notably pathogenicity determinants $[3,46,59]$. Only 4 putative GEIs were identified in strain 

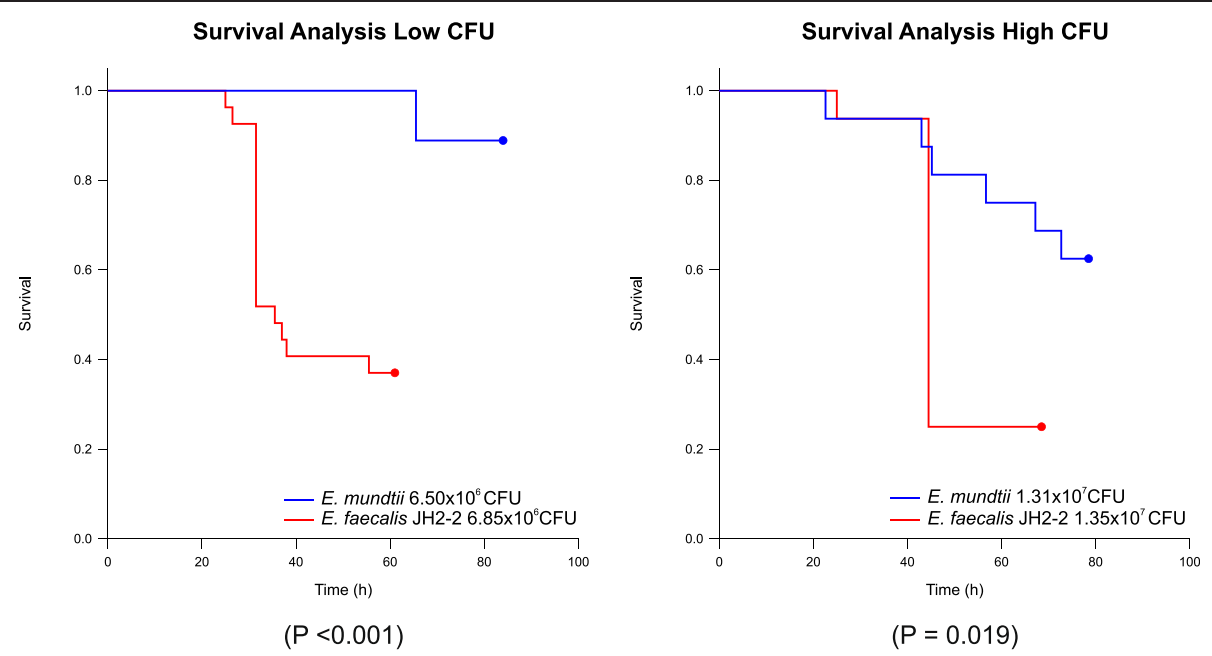

Figure 3 E. mundtii effect on G. mellonella survival. Kaplan-Meier survival analysis of G. mellonella upon infection with E. mundtii (blue lines) and E. faecalis JH2-2 (red lines). Data are representative of three separate survival experiments performed with groups of 15 insects for each. Survival curves were constructed by the Kaplan-Meier method and compared by Log-rank analysis. P values of $<0.05$ were considered statistically significant.

CRL1656, and none of them carries genes that might be encoding known virulence determinants. Many virulence genes and their functions have been described in enterococci $[3,46,59]$. Our bioinformatic analysis revealed the lowest number of putative pathogenicity factors for E. mundtii in comparison to other Enterococcus strains (Table 1), including different species from diverse origins (clinical, food and commensal). However the analysis of the presence or absence of virulence traits is not decisive to determine the commensal or pathogenic nature of a bacterium and the predictive methods have limitations and are not sufficient to conclude on the pathogenesis of the microorganism. This observation clearly arises from the analysis of Table 1, in which the commensal strain E. faecium Com 15 possesses a higher number of putative virulence determinants (755) than the clinical isolate E. faecium strain DO (728). In this study, the virulence of the E. mundtii CRL1665 strain was evaluated using the G. mellonela model. As shown in the Figure 3, E. mundtii CRL1665 resulted non-virulent at low dose whereas the JH2-2 strain used as control killed $60 \%$ of larvae at $60 \mathrm{~h}$. At high dose similar results were found E. mundtii strain was able to kill only $40 \%$ of larvae, whereas the control killed near the $80 \%$. Our data indicate that the E mundtii strain CRL1665 as it was predicted by bioinformatic analysis is less virulent than the model E. faecalis JH2-2.

Remarkably, neither homologues for the main secreted factors shown to be critical for enterococcal pathogenesis, sprE (serine protease) and gelE (gelatinase), were found in $E$. mundtii nor other relevant genes such as $c y l$ (transport and activation of a cytolisin [69]), agg, (adherence to eukaryotic cells) and esp (cell wall protein involved in immune evasion [70]). Interestingly, a gene annotated as a putative hemolysin was found. This protein contains a domain belonging to the hemolysin III family (Pfam 03006), which includes proteins from pathogenic and non-pathogenic bacteria, Homo sapiens and Drosophila melanogaster. In Bacillus cereus, it has been shown to function as a channel-forming cytolysin. In contrast, the cytolytic hemolysins commonly expressed by many enterococcal bacteraemia isolates, are two-component peptide systems $\left(\mathrm{CylL}_{\mathrm{L}}\right.$ and $\left.\mathrm{CylL}_{\mathrm{S}}\right)$ whose expression requires the products of an eigth-gene locus [71]. However, this locus is absent in E. mundtii. Experimental assays will be needed to define if the type III-hemolysin has a lytic role in vivo.

Some enterococcal species, particularly those thought to be associated with soil and non-human hosts [72], including E. mundtii, contain yellow pigments. Pigments in E. mundtii were identified as carotenoids [73]. This study has shown the presence of genes involved in the C30 carotenoid biosynthetic pathway in this bacterium, which is also present in most of the environmental enterococcal sequenced species. The ubiquitous detection of $\operatorname{crt} M$ and $\operatorname{crt} N$ genes, involved in the biosynthesis of the yellow 4,4'-diaponeurosporene, as well as the documented carotenoid production by these microorganisms [4], suggest that the role of carotenoids in enterococcal environmental fitness must be important. Carotenoids function as protectors against photodamage as they are able to quench ROS. Pigmented enterococci are protected from solar inactivation and can persist for extended periods of time in marine water relative to nonpigmented species [56]. Therefore, we can speculate that pigmentation in E. mundtii could play a protective role when the microorganism is exposed to the environment.

In this work we also identified those genes encoding the machinery responsible for the production of a 
bacteriocin previously reported by Espeche et al. [8]. This feature constitutes a relevant and unique characteristic of the strain. With respect to its biological activity, mundticin 1656 was shown to inhibit the growth of Listeria monocytogenes and a variety of lactic acid bacteria [8]. Noteworthy, the use of bacteriocins for the prevention or treatment of mastitis in cows has the potential to reduce the dependence on antibiotics. Given the genetic features observed throughout this work, E. mundtii could be used to this end, but this hypothesis needs to be further investigated.

\section{Additional files}

Additional file 1: Figure S1. Search for orthologs of E. mundtii CRL1656 putative regulators in E. faecalis $\mathrm{V} 583$ and E. faecium DO genomes. A) General workflow. E. mundtii CRL1656 regulators were used as query in a BlastP search over E. faecalis V583 and E. faecium DO genomes. Obtained data was used to define Cut-In and Cut-Off values and subsequently to determine the presence or absence of each regulator in both genomes. B) Construction of a Correlation Table. Retrieved sequences from previous BlastP analysis with more than $80 \%$ coverage, score values higher than 50 and sharing the highest degree of identity were directly added to the Table. C) Workflow for Cut-In and Cut-Off definition. E. faecalis V583 or E. faecium DO proteins correlating with more than one E. mundtii CRL1656 protein were used as Input Data. Each set of E. mundtii CRL1656 proteins was sorted by percentage of identity (Id\%). Those with the highest value and more than $50 \%$ of Id\% were defined as orthologs and used for Cut-In definition. The remaining proteins were defined as paralogs and used to set up the Cut-Off value. Protein sets with the highest Id\% but lower than 50\% were analyzed individually to determine whether they were paralogs or orthologs. Finally, the lowest Id\% value among all orthologs was defined as the Cut-In value and the highest Id\% among all the paralogs was defined as Cut-Off value. D) Workflow for Ortholog Table construction. The pruned correlation table results from eliminating paralogs defined in (C) from the correlation table defined in (B). All proteins that shared an Id\% higher than the Cut-In value were considered as present and those with Id\% lower than the Cut-Off were considered absent. Presence or absence of proteins with shared identity between Cut-Off and Cut-In values was analyzed individually by sinteny.

Additional file 2: Table S1. Putative functions associated to horizonta gene transfer.

Additional file 3: Table S2. Transcriptional regulators encoded in the E. mundtii genome.

Additional file 4: Table S3. Two-component systems present in E. mundtii.

Additional file 5: Figure S2. epa and eps gene clusters present in E. mundtti CRL1656. E. faecium 1.141 .733 epa genes and E. faecium Com 15 eps genes are shown for comparison. Genes are colored following the code included in the figure.

Additional file 6: Table S4. Eps and epa loci in E. mundtii.

Additional file 7: Table S5. Antibiotic resistance.

\section{Competing interests}

Authors declare that they have no competing interests.

\section{Authors' contributions}

GDR, ME, VSB, CAS and LE conducted bioinformatic analysis and G. mellonella killing assays. CM designed the study and drafted the manuscript. All authors were involved in the writing of the manuscript. All authors read and approved the final manuscript.

\section{Acknowledgements}

This work was supported by grants from the Agencia Nacional de Promoción Científica y Tecnológica (ANPCyT, PICT2010-1828 and PIP 2012/14 CONICET, Argentina). GDR and CAS are fellows of CONICET, VSB, ME and CM are Career Investigators of the same institution.

\section{Author details}

${ }^{1}$ Instituto de Biología Molecular y Celular de Rosario (IBR-CONICET) and Departamento de Microbiología, Facultad de Ciencias Bioquímicas y Farmacéuticas, Universidad Nacional de Rosario, Suipacha 531, Rosario S2002LRK, Argentina. ${ }^{2}$ Departamento de Fisiología, Facultad de Ciencias Médicas, Universidad Nacional de Rosario, Santa Fe 3100, Rosario S2002LRK Argentina.

Received: 1 October 2013 Accepted: 2 June 2014 Published: 18 June 2014

References

1. Giraffa G: Functionality of Enterococci in dairy products. Int J Food Microbiol 2003, 88(2-3):215-222.

2. Ogier JC, Serror P: Safety assessment of dairy microorganisms: the Enterococcus genus. Int J Food Microbiol 2008, 126(3):291-301.

3. Arias CA, Murray BE: The rise of the Enterococcus: beyond vancomycin resistance. Nat Rev Microbiol 2012, 10(4):266-278.

4. Collins MD, Farrow JAE, Jones D: Enterococcus mundtii sp. nov. Int J Syst Bacteriol 1986, 36(1):8-12

5. Muller T, Ulrich A, Ott EM, Muller M: Identification of plant-associated enterococci. J Appl Microbiol 2001, 91(5):268-278.

6. Higashide T, Takahashi M, Kobayashi A, Ohkubo S, Sakurai M, Shirao Y, Tamura T, Sugiyama K: Endophthalmitis caused by Enterococcus mundtii. J Clin Microbiol 2005, 43(3):1475-1476.

7. Magni C, Espeche C, Repizo GD, Saavedra L, Suarez CA, Blancato VS, Espariz M, Esteban L, Raya RR, de Font Valdez G, Vignolo G, Mozzi F, Taranto MP, Hebert EM, Nader-Macias ME, Sesma F: Draft genome sequence of Enterococcus mundtii CRL1656. J Bacteriol 2012, 194(2):550.

8. Espeche MC, Otero MC, Sesma F, Nader-Macias ME: Screening of surface properties and antagonistic substances production by lactic acid bacteria isolated from the mammary gland of healthy and mastitic cows. Vet Microbiol 2009, 135(3-4):346-357.

9. Aziz RK, Bartels D, Best AA, DeJongh M, Disz T, Edwards RA, Formsma K, Gerdes S, Glass EM, Kubal M, Meyer F, Olsen GJ, Olson R, Osterman AL, Overbeek RA, McNeil LK, Paarmann D, Paczian T, Parrello B, Pusch GD, Reich C, Stevens R, Vassieva O, Vonstein V, Wilke A, Zagnitko O: The RAST Server: rapid annotations using subsystems technology. BMC Genomics 2008, 9:75

10. Paulsen IT, Banerjei L, Myers GS, Nelson KE, Seshadri R, Read TD, Fouts DE, Eisen JA, Gill SR, Heidelberg JF, Tettelin H, Dodson RJ, Umayam L, Brinkac L, Beanan M, Daugherty S, DeBoy RT, Durkin S, Kolonay J, Madupu R, Nelson W, Vamathevan J, Tran B, Upton J, Hansen T, Shetty J, Khouri H, Utterback T, Radune D, Ketchum KA, Dougherty BA, Fraser CM: Role of mobile DNA in the evolution of vancomycin-resistant Enterococcus faecalis. Science 2003, 299(5615):2071-2074.

11. van Schaik W, Top J, Riley DR, Boekhorst J, Vrijenhoek JE, Schapendonk CM, Hendrickx AP, Nijman IJ, Bonten MJ, Tettelin H, Willems RJ:

Pyrosequencing-based comparative genome analysis of the nosocomial pathogen Enterococcus faecium and identification of a large transferable pathogenicity island. BMC Genomics 2010, 11:239.

12. Brede DA, Snipen LG, Ussery DW, Nederbragt AJ, Nes IF: Complete genome sequence of the commensal Enterococcus faecalis 62, isolated from a healthy Norwegian infant. J Bacterio/ 2011, 193(9):2377-2378.

13. Qin X, Galloway-Pena JR, Sillanpaa J, Roh JH, Nallapareddy SR, Chowdhury S, Bourgogne A, Choudhury T, Muzny DM, Buhay CJ, Ding Y, Dugan-Rocha S, Liu W, Kovar C, Sodergren E, Highlander S, Petrosino JF, Worley KC, Gibbs RA, Weinstock GM, Murray BE: Complete genome sequence of Enterococcus faecium strain TX16 and comparative genomic analysis of Enterococcus faecium genomes. BMC Microbiol 2012, 12:135.

14. McBride SM, Fischetti VA, Leblanc DJ, Moellering RC Jr, Gilmore MS: Genetic diversity among Enterococcus faecalis. PLoS One 2007, 2(7):e582.

15. Fortina MG, Ricci G, Mora D, Manachini PL: Molecular analysis of artisanal Italian cheeses reveals Enterococcus italicus sp. nov. Int J Syst Evol Microbiol 2004, 54(Pt 5):1717-1721. 
16. Palmer KL, Godfrey P, Griggs A, Kos VN, Zucker J, Desjardins C, Cerqueira G, Gevers D, Walker S, Wortman J, Feldgarden M, Haas B, Birren B, Gilmore MS: Comparative genomics of enterococci: variation in Enterococcus faecalis clade structure in E. faecium, and defining characteristics of $\mathrm{E}$. gallinarum and E. casseliflavus. mBio 2012, 3(1):e00318-00311.

17. Richter M, Rossello-Mora R: Shifting the genomic gold standard for the prokaryotic species definition. Proc Natl Acad Sci U S A 2009, 106(45):19126-19131.

18. Goris J, Konstantinidis KT, Klappenbach JA, Coenye T, Vandamme P, Tiedje JM: DNA-DNA hybridization values and their relationship to whole-genome sequence similarities. Int J Syst Evol Microbio/ 2007, 57(Pt 1):81-91.

19. Vernikos GS, Parkhill J: Interpolated variable order motifs for identification of horizontally acquired DNA: revisiting the Salmonella pathogenicity islands. Bioinformatics 2006, 22(18):2196-2203.

20. Waack S, Keller O, Asper R, Brodag T, Damm C, Fricke WF, Surovcik K, Meinicke $P$, Merkl R: Score-based prediction of genomic islands in prokaryotic genomes using hidden Markov models. BMC bioinformatics 2006, 7:142

21. Langille MG, Hsiao WW, Brinkman FS: Evaluation of genomic island predictors using a comparative genomics approach. BMC bioinformatics 2008, 9:329.

22. Rutherford K, Parkhill J, Crook J, Horsnell T, Rice P, Rajandream MA, Barrell B: Artemis: sequence visualization and annotation. Bioinformatics 2000 16(10):944-945.

23. Juhas M, van der Meer JR, Gaillard M, Harding RM, Hood DW, Crook DW: Genomic islands: tools of bacterial horizontal gene transfer and evolution. FEMS Microbiol Rev 2009, 33(2):376-393.

24. Zhou CE, Smith J, Lam M, Zemla A, Dyer MD, Slezak T: MvirDB-a microbial database of protein toxins, virulence factors and antibiotic resistance genes for bio-defence applications. Nucleic Acids Res 2007, 35(Database issue):D391-394.

25. Chen F, Mackey AJ, Stoeckert CJ Jr, Roos DS: OrthoMCL-DB: querying a comprehensive multi-species collection of ortholog groups. Nucleic Acids Res 2006, 34(Database issue):D363-368.

26. Larkin MA, Blackshields G, Brown NP, Chenna R, McGettigan PA, McWilliam H, Valentin F, Wallace IM, Wilm A, Lopez R, Thompson JD, Gibson TJ, Higgins DG Clustal W and Clustal X version 2.0. Bioinformatics 2007, 23(21):2947-2948.

27. Talavera G, Castresana J: Improvement of phylogenies after removing divergent and ambiguously aligned blocks from protein sequence alignments. Syst Biol 2007, 56(4):564-577.

28. Stamatakis A, Hoover $P$, Rougemont J: A rapid bootstrap algorithm for the RAxML Web servers. Syst Biol 2008, 57(5):758-771.

29. Lebreton F, Riboulet-Bisson E, Serror P, Sanguinetti M, Posteraro B, Torelli $R$, Hartke A, Auffray Y, Giard JC: Ace, which encodes an adhesin in Enterococcus faecalis, is regulated by Ers and is involved in virulence. Infect Immun 2009, 77(7):2832-2839.

30. Konstantinidis KT, Tiedje JM: Genomic insights that advance the species definition for prokaryotes. Proc Natl Acad Sci U S A 2005, 102(7):2567-2572.

31. Kawamoto S, Shima J, Sato R, Equchi T, Ohmomo S, Shibato J, Horikoshi N, Takeshita K, Sameshima T: Biochemical and genetic characterization of mundticin KS, an antilisterial peptide produced by Enterococcus mundtii NFRI 7393. Appl Environ Microbiol 2002, 68(8):3830-3840.

32. Bennik MH, Vanloo B, Brasseur R, Gorris LG, Smid EJ: A novel bacteriocin with a YGNGV motif from vegetable-associated Enterococcus mundtii: full characterization and interaction with target organisms. Biochim Biophys Acta 1998, 1373(1):47-58

33. Drider D, Fimland G, Hechard Y, McMullen LM, Prevost H: The continuing story of class Ila bacteriocins. Microbiol Mol Biol Rev 2006, 70(2):564-582.

34. Cotter PD, Hill C: Surviving the acid test: responses of gram-positive bacteria to low pH. Microbiol Mol Biol Rev 2003, 67(3):429-453. table of contents.

35. Krulwich TA, Sachs G, Padan E: Molecular aspects of bacterial pH sensing and homeostasis. Nat Rev Microbiol 2011, 9(5):330-343.

36. Blancato VS, Repizo GD, Suarez CA, Magni C: Transcriptional regulation of the citrate gene cluster of Enterococcus faecalis Involves the GntR family transcriptional activator CitO. J Bacteriol 2008, 190(22):7419-7430.

37. Repizo GD, Blancato VS, Mortera P, Lolkema JS, Magni C: Biochemical and genetic characterization of the Enterococcus faecalis oxaloacetate decarboxylase complex. App/ Environ Microbiol 2013, 79(9):2882-2890.

38. Suarez CA, Blancato VS, Poncet S, Deutscher J, Magni C: CcpA represses the expression of the divergent cit operons of Enterococcus faecalis through multiple cre sites. BMC Microbiol 2011, 11:227
39. Espariz M, Repizo G, Blancato V, Mortera P, Alarcon S, Magni C: Identification of malic and soluble oxaloacetate decarboxylase enzymes in Enterococcus faecalis. FEBS J 2011, 278(12):2140-2151.

40. Mortera P, Espariz M, Suarez C, Repizo G, Deutscher J, Alarcon S, Blancato V, Magni C: Fine-tuned transcriptional regulation of malate operons in Enterococcus faecalis. Appl Environ Microbiol 2012, 78(6):1936-1945.

41. Sillanpaa J, Nallapareddy SR, Prakash VP, Qin X, Hook M, Weinstock GM, Murray BE: Identification and phenotypic characterization of a second collagen adhesin, $\mathrm{Scm}$, and genome-based identification and analysis of 13 other predicted MSCRAMMs, including four distinct pilus loci, in Enterococcus faecium. Microbiology 2008, 154(Pt 10):3199-3211.

42. Nallapareddy SR, Singh KV, Sillanpaa J, Garsin DA, Hook M, Erlandsen SL, Murray BE: Endocarditis and biofilm-associated pili of Enterococcus faecalis. J Clin Invest 2006, 116(10):2799-2807.

43. Bourgogne A, Singh KV, Fox KA, Pflughoeft KJ, Murray BE, Garsin DA: EbpR is important for biofilm formation by activating expression of the endocarditis and biofilm-associated pilus operon (ebpABC) of Enterococcus faecalis OG1RF. J Bacteriol 2007, 189(17):6490-6493.

44. Guiton PS, Hung CS, Hancock LE, Caparon MG, Hultgren SJ: Enterococcal biofilm formation and virulence in an optimized murine model of foreign body-associated urinary tract infections. Infect Immun 2010, 78(10):4166-4175.

45. Gao P, Pinkston KL, Nallapareddy SR, van Hoof A, Murray BE, Harvey BR: Enterococcus faecalis $r n j B$ is required for pilin gene expression and biofilm formation. J Bacteriol 2010, 192(20):5489-5498.

46. Rigottier-Gois L, Alberti A, Houel A, Taly JF, Palcy P, Manson J, Pinto D, Matos RC, Carrilero L, Montero N, Tariq M, Karsens H, Repp C, Kropec A, Budin-Verneuil A, Benachour A, Sauvageot N, Bizzini A, Gilmore MS, Bessieres P, Kok J, Huebner J, Lopes F, Gonzalez-Zorn B, Hartke A, Serror P: Large-scale screening of a targeted Enterococcus faecalis mutant library identifies envelope fitness factors. PLoS One 2011, 6(12):e29023.

47. Theilacker C, Sava I, Sanchez-Carballo P, Bao Y, Kropec A, Grohmann E, Holst $\mathrm{O}$, Huebner J: Deletion of the glycosyltransferase bgsB of Enterococcus faecalis leads to a complete loss of glycolipids from the cell membrane and to impaired biofilm formation. BMC Microbiol 2011, 11:67.

48. Hufnagel M, Koch S, Creti R, Baldassarri L, Huebner J: A putative sugarbinding transcriptional regulator in a novel gene locus in Enterococcus faecalis contributes to production of biofilm and prolonged bacteremia in mice. J Infect Dis 2004, 189(3):420-430.

49. Teng F, Singh KV, Bourgogne A, Zeng J, Murray BE: Further characterization of the epa gene cluster and Epa polysaccharides of Enterococcus faecalis. Infect Immun 2009, 77(9):3759-3767.

50. Teng F, Kawalec M, Weinstock GM, Hryniewicz W, Murray BE: An Enterococcus faecium secreted antigen, SagA, exhibits broad-spectrum binding to extracellular matrix proteins and appears essential for $E$. faecium growth. Infect Immun 2003, 71(9):5033-5041.

51. Zhao C, Hartke A, La Sorda M, Posteraro B, Laplace JM, Auffray $Y$ Sanguinetti M: Role of methionine sulfoxide reductases $A$ and $B$ of Enterococcus faecalis in oxidative stress and virulence. Infect Immun 2010, 78(9):3889-3897.

52. Choudhury T, Singh KV, Sillanpaa J, Nallapareddy SR, Murray BE: Importance of two Enterococcus faecium loci encoding Gls-like proteins for in vitro bile salts stress response and virulence. J Infect Dis 2011, 203(8):1147-1154.

53. Le Jeune A, Torelli R, Sanguinetti M, Giard JC, Hartke A, Auffray Y, Benachour A: The extracytoplasmic function sigma factor SigV plays a key role in the original model of lysozyme resistance and virulence of Enterococcus faecalis. PLoS One 2010, 5(3):e9658.

54. Frank KL, Barnes AM, Grindle SM, Manias DA, Schlievert PM, Dunny GM: Use of recombinase-based in vivo expression technology to characterize Enterococcus faecalis gene expression during infection identifies in vivo-expressed antisense RNAs and implicates the protease Eep in pathogenesis. Infect Immun 2012, 80(2):539-549.

55. Hancock LE, Perego M: Systematic inactivation and phenotypic characterization of two-component signal transduction systems of Enterococcus faecalis V583. J Bacteriol 2004, 186(23):7951-7958.

56. Maraccini PA, Ferguson DM, Boehm AB: Diurnal variation in Enterococcus species composition in polluted ocean water and a potential role for the enterococcal carotenoid in protection against photoinactivation. Appl Environ Microbiol 2012, 78(2):305-310.

57. Pelz A, Wieland KP, Putzbach K, Hentschel P, Albert K, Gotz F: Structure and biosynthesis of staphyloxanthin from Staphylococcus aureus. J Biol Chem 2005, 280(37):32493-32498. 
58. Garrido-Fernandez J, Maldonado-Barragan A, Caballero-Guerrero B, Hornero-Mendez D, Ruiz-Barba JL: Carotenoid production in Lactobacillus plantarum. Int J Food Microbiol 2010, 140(1):34-39.

59. Hanin A, Sava I, Bao Y, Huebner J, Hartke A, Auffray Y, Sauvageot N: Screening of in vivo activated genes in Enterococcus faecalis during insect and mouse infections and growth in urine. PLoS One 2010, 5(7):e11879.

60. Bourgogne A, Thomson LC, Murray BE: Bicarbonate enhances expression of the endocarditis and biofilm associated pilus locus, ebpR-ebpABC, in Enterococcus faecalis. BMC Microbiol 2010, 10:17.

61. Tendolkar PM, Baghdayan AS, Shankar N: Putative surface proteins encoded within a novel transferable locus confer a high-biofilm phenotype to Enterococcus faecalis. J Bacteriol 2006, 188(6):2063-2072.

62. Lebreton F, Le Bras F, Reffuveille F, Ladjouzi R, Giard JC, Leclerca R, Cattoir V: Galleria mellonella as a model for studying Enterococcus faecium host persistence. J Mol Microbiol Biotechnol 2011, 21(3-4):191-196.

63. Bergin D, Reeves EP, Renwick J, Wientjes FB, Kavanagh K: Superoxide production in Galleria mellonella hemocytes: identification of proteins homologous to the NADPH oxidase complex of human neutrophils. Infect Immun 2005, 73(7):4161-4170.

64. Jacob AE, Hobbs SJ: Conjugal transfer of plasmid-borne multiple antibiotic resistance in Streptococcus faecalis var. zymogenes. J Bacterio/ 1974, 117(2):360-372.

65. Suarez C, Espariz M, Blancato VS, Magni C: Expression of the agmatine deiminase pathway in Enterococcus faecalis Is Activated by the AguR Regulator and Repressed by CcpA and PTS(Man) Systems. PLoS One 2013, 8(10):e76170

66. Bizzini A, Zhao C, Auffray Y, Hartke A: The Enterococcus faecalis superoxide dismutase is essential for its tolerance to vancomycin and penicillin. J Antimicrob Chemother 2009, 64(6):1196-1202.

67. Benachour A, Ladjouzi R, Le Jeune A, Hebert L, Thorpe S, Courtin P, Chapot-Chartier MP, Prajsnar TK, Foster SJ, Mesnage S: The lysozyme-induced peptidoglycan N-acetylglucosamine deacetylase PgdA (EF1843) is required for Enterococcus faecalis virulence. J Bacteriol 2012, 194(22):6066-6073.

68. Poyart C, Quesnes G, Trieu-Cuot P: Sequencing the gene encoding manganese-dependent superoxide dismutase for rapid species identification of Enterococci. J Clin Microbiol 2000, 38(1):415-418.

69. Kayser FH: Safety aspects of Enterococci from the medical point of view. Int J Food Microbiol 2003, 88:255-262.

70. Sava IG, Heikens E, Huebner J: Pathogenesis and immunity in Enterococcal infections. Clin Microbiol Infect 2010, 16(6):533-540.

71. Cox CR, Coburn PS, Gilmore MS: Enterococcal cytolysin: a novel two component peptide system that serves as a bacterial defense against eukaryotic and prokaryotic cells. Curr Protein Pept Sci 2005, 6(1):77-84.

72. Bahirathan M, Puente L, Seyfried P: Use of yellow-pigmented Enterococci as a specific indicator of human and nonhuman sources of faecal pollution. Can J Microbiol 1998, 44(11):1066-1071.

73. Breithaupt DE, Bamedi A: Carotenoid esters in vegetables and fruits: a screening with emphasis on beta-cryptoxanthin esters. J Agric Food Chem 2001, 49(4):2064-2070.

doi:10.1186/1471-2164-15-489

Cite this article as: Repizo et al:: Genomic comparative analysis of the environmental Enterococcus mundtii against enterococcal representative species. BMC Genomics 2014 15:489

\section{Submit your next manuscript to BioMed Central and take full advantage of:}

- Convenient online submission

- Thorough peer review

- No space constraints or color figure charges

- Immediate publication on acceptance

- Inclusion in PubMed, CAS, Scopus and Google Scholar

- Research which is freely available for redistribution 